ICRDET-2021, February 26-27, 2021, AICE, Jaipur, India

International Journal of Technical Research \& Science (Special Issue) ISSN No.:2454-2024 (online)

\title{
ELECTRONIC PANIC ALARM SYSTEM
}

\author{
Vineet Chhabra, Shambhu Dayal Sharma, Rakshita Singhal \\ E-Mail Id: vineet.chhabra@anandice.ac.in \\ Department of Electronics \& Communication, Anand International College of Engineering, Jaipur, \\ Rajasthan (India)
}

Abstract-Health issues are very common nowadays due to our lifestyle. In some of the conditions we can't even help ourselves in that situation like a panic attack or something. In that situation if we need help we need a more easier way to find help, this device does the same for us. In this paper we will discuss all about the device, how it works, what are the uses of it etc.

Keywords-Arduino, UNO, Potentiometer, Microcontroller.

\section{INTRODUCTION}

It is not easy to live alone when you live alone and have some health issues at the same time. You need someone who can help you in the same and have your back. So when you get sick you need someone to take care of you and in a serious health issue which can happen any time you need the help as soon as possible, so in that situation you need to contact someone who can help you, for that you need some device which is easy to operate. This device is for the same.You just need to press a button to contact someone. The button will be located on your wrist watch, you just have to press it and it will send a scripted message to your selected contacts along with your location with the help of your mobile phone.Now let's talk about its background or past models. In the past models of this device only the location is sent and they are more costly than this. In the past models its not connected to your mobile phone or any external device that's why it was so expensive. But in this one I've tried to make it less expensive and affordable. The organization of this paper is as follows: in the second section the methodology is discussed. Then in section three the design and used components are discussed. Then the used software and then the conclusion. In the last section references are mentioned.

\section{METHODOLOGY}

\subsection{Working Method}

In this project we have used Arduino UNO, 16*2 LCD, push button, DS1307, Bluetooth module (HC-05), potentiometer, breadboard, Embedded $\mathrm{C}++$, mobile application. When you press the button on your wrist watch, which is connected to your mobile application with the help of Bluetooth, it will navigate the signal to the mobile application and then the mobile application will send a scripted message to your selected contacts along with your location. The LCD displays the real time and date and when the button is pressed the LCD shows a message that "button pressed". The action on the hardware device or we can say the wrist watch will be reflected on the mobile application. The mobile application and the hardware is connected through Bluetooth. This whole project works on Bluetooth technology. The circuit is just the representation so the final design would be of customized components.

\subsection{Components}

\subsubsection{LCD display}

The term LCD stands for liquid crystal display. It is one kind of electronic display module used in an extensive range of applications like various circuits $\&$ devices like mobile phones, calculators, computers, TV sets, etc. These displays are mainly preferred for multi-segment light-emitting diodes and seven segments. The main benefits of using this module are inexpensive; simply programmable, animations, and there are no limitations for displaying custom characters, special and even animations, etc.

\subsubsection{Arduino UNO}

Arduino/Genuino Uno is a microcontroller board based on the ATmega328P (datasheet). It has 14 digital input/output pins (of which 6 can be used as PWM outputs), 6 analog inputs, a $16 \mathrm{MHz}$ quartz crystal, a USB connection, a power jack, an ICSP header and a reset button. It contains everything needed to support the microcontroller; simply connect it to a computer with a USB cable or power it with a AC-to-DC adapter or battery to get started.. You can tinker with your UNO without working too much about doing something wrong, worst case scenario you can replace the chip for a few dollars and start over again.

\subsubsection{DS1307}

The DS1307 serial real-time clock (RTC) is a low-power, full binary-coded decimal (BCD) clock/calendar plus 56 bytes of NV SRAM. Address and data are transferred serially through an I2C, bidirectional bus. The clock/calendar provides seconds, minutes, hours, day, date, month, and year information. The end of the month date is automatically adjusted for months with fewer than 31 days, including corrections for leap year. The clock operates in either the 24-hour or 12-hour format with AM/PM indicator. The DS1307 has a built-in power-sense circuit that detects power failures and automatically switches to the backup supply. Timekeeping operation continues while the part operates from the backup supply.

\subsubsection{Push button}

A push-button (also spelled pushbutton) or simply button is a simple switch mechanism to control some aspect of a machine or a process. Buttons are typically made out of hard material, usually plastic or metal.[1] The 
ICRDET-2021, February 26-27, 2021, AICE, Jaipur, India

International Journal of Technical Research \& Science (Special Issue) ISSN No.:2454-2024 (online)

surface is usually flat or shaped to accommodate the human finger or hand, so as to be easily depressed or pushed. Buttons are most often biased switches, although many unbiased buttons (due to their physical nature) still require a spring to return to their unpushed state. Terms for the "pushing" of a button include pressing, depressing, mashing, slapping, hitting, and punching.

\subsubsection{Bluetooth}

Wireless communication is swiftly replacing the wired connection when it comes to electronics and communication. Designed to replace cable connections, HC-05 uses serial communication to communicate with the electronics. Usually, it is used to connect small devices like mobile phones using a short-range wireless connection to exchange files. It uses the $2.45 \mathrm{GHz}$ frequency band. The transfer rate of the data can vary up to $1 \mathrm{Mbps}$ and is in range of 10 meters. The HC- 05 module can be operated within 4-6V of power supply. It supports baud rate of 9600, 19200, 38400, 57600, etc. Most importantly it can be operated in Master-Slave mode which means it will neither send or receive data from external sources.

\subsubsection{Potentiometer}

A potentiometer is a three-terminal resistor with a sliding or rotating contact that forms an adjustable voltage divider. If only two terminals are used, one end and the wiper, it acts as a variable resistor or rheostat. The measuring instrument called a potentiometer is essentially a voltage divider used for measuring electric potential (voltage); the component is an implementation of the same principle, hence its name.

Potentiometers are commonly used to control electrical devices such as volume controls on audio equipment. Potentiometers operated by a mechanism can be used as position transducers, for example, in a joystick. Potentiometers are rarely used to directly control significant power (more than a watt), since the power dissipated in the potentiometer would be comparable to the power in the controlled load.

\section{SOFTWARE}

\subsection{Arduino IDE}

The Arduino Integrated Development Environment (IDE) is a cross-platform application (for Windows, macOS, Linux) that is written in functions from $\mathrm{C}$ and $\mathrm{C}++$. It is used to write and upload programs to Arduino compatible boards, but also, with the help of third-party cores, other vendor development boards.

The source code for the IDE is released under the GNU General Public License, version 2 The Arduino IDE supports the languages $\mathrm{C}$ and $\mathrm{C}++$ using special rules of code structuring.[6] The Arduino IDE supplies a software library from the Wiring project, which provides many common input and output procedures. Userwritten code only requires two basic functions, for starting the sketch and the main program loop, that are compiled and linked with a program stub main() into an executable cyclic executive program with the GNU toolchain, also included with the IDE distribution. The Arduino IDE employs the program avrdude to convert the executable code into a text file in hexadecimal encoding that is loaded into the Arduino board by a loader program in the board's firmware. By default, avrdude is used as the uploading tool to flash the user code onto official Arduino boards. Arduino IDE is a derivative of the Processing IDE, however as of version 2.0, the Processing IDE will be replaced with the Visual Studio Code-based Eclipse The IDE framework.

With the rising popularity of Arduino as a software platform, other vendors started to implement custom open source compilers and tools (cores) that can build and upload sketches to other microcontrollers that are not supported by Arduino's official line of microcontrollers.

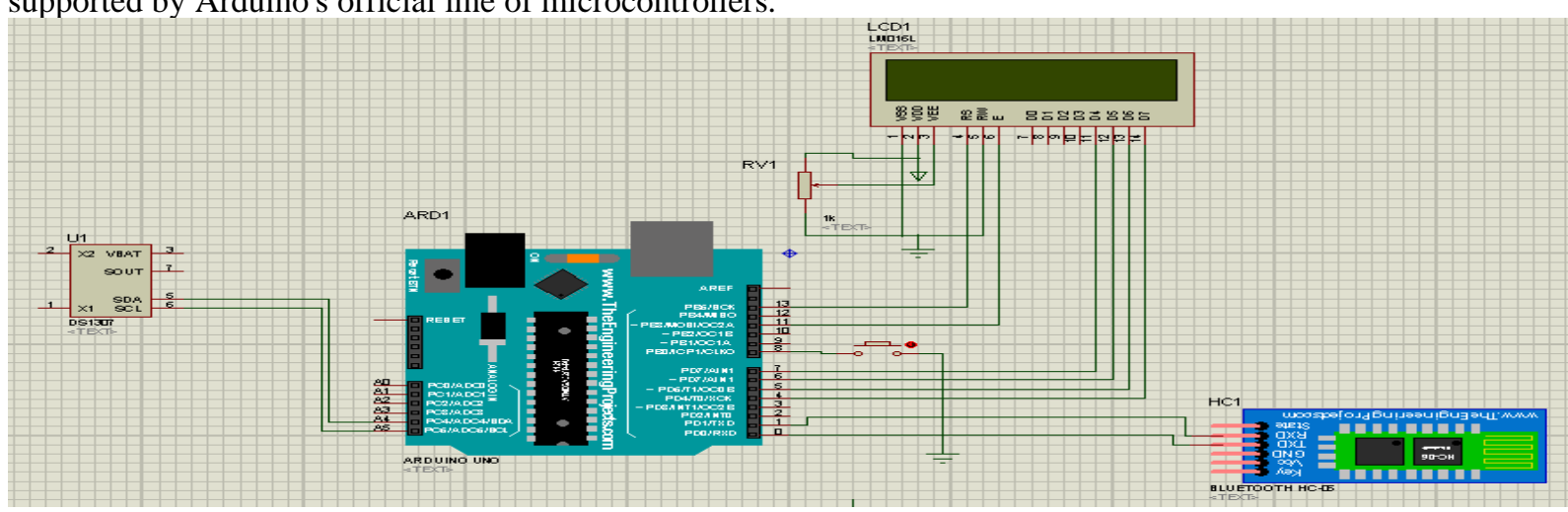

Fig. 3.1 Circuit Design

\section{CONCLUSION}

The project is made to get help in any health issue when its hard to handle yourself. It can be implemented in any health situation because it's a wrist watch. The targeted audiences are people with a health issue (an adult). It won't be connected to the authority so the control is in your hands. It can save lives.

\section{REFERENCES}

[1] Programming Arduino: Getting Started With Sketches (second edition), (2017).

[2] Arduino Workshop: A Hands-On Introduction with 65 Projects 1st Edition (2015). Arduino: Advanced Methods and Strategies of Using Arduino

[3] Arduino Programming: The Ultimate Guide For Making The Best Of Your Arduino Programming Projects. 